\title{
CARACTERÍSTICAS SEMINAIS DE CÃES dA RAÇA PASTOR ALEMÃo
}

\author{
SEMINAL CHARACTERISTICS OF GERMAN SHEPHERD DOC;S
}

\author{
José Adalmir 'Torres de SOUKA !: Walter SPICCIATI2; José Antônio VISINTIN2: Valquiria Hyppólito BARXABE, 3;
} Renato Campanarut BARNABE?

\begin{abstract}
RESUMO
Sêmen de 25 cães da raça Pastor Alemão, com 1 a 7 anos de idade e pesando 30 a 3.5 quilos foi coletado por masturbação. Com o objetivo de utilizar o material para inseminação artificial, colheu-se a segunda e parte dat terceira fração, constatandose. em média. volume de $7.17 \mathrm{ml}$ : cor brancia e aspecto leitoso: motilidade de $68.84 \%$; vigor de 3.5 ; concentração de 136.192 espermatuzóides por $\mathrm{mm}^{3}$ e de $889.772 .00 \%$ por ejaculado; defeitos espermáticos maiores de $9.92 \%$ e menores de $7,62 \%$. 0 pH do sêmen, dos diluidores e do sêmen diluído variou. respectivamente, de 6.02 a 6.60; 5,85 a 6.90; c 5.97 a 6,76; a pressāo osmótica de 285.20 a 295.00):240.00 a 310.00): e 272.32 a 303.92 mOsmois e a concentração de sódio e potássio, respectivamente. para o plasma seminal e os 4 diluidores, de 134.40 a 156,$00 ; 7,80$ a $13.72 ; 3,00$ a 270.00$)$; e 6,00 a $25,00 \mathrm{mF}: \mathrm{q} / \mathrm{l}$. Verificou-se que entre cães. houve variação significante de todas as características seminais, exceto al pressão osmótica e. ainda. correlaçoès entre os caracteres seminais e entre estes e os totais de patologias espermáticass. A varriabilidade das características seminais entre os cāes foi hem maior quando comparada entre as colheitas de cada animal. exceto a pressão osmótica e a concentração de sódio, yue se apresentaram de maneira inversa.
\end{abstract}

UNITERMos: Sêmen: Cães da raça Pastor Alemão

\section{INTRODUÇÃO}

Na espécie canina, o método da masturbação é preferível por permitir uma individualização das diferentes frações do ejaculado, propiciando ainda o estudo perfeito das características seminais!, evitando efeitos deletérios sobre os espermatozóides 12 .

As características seminais relacionadas ao volume, concentração. motilidade e $\mathrm{pH}$ têm sido estudadas na espécic canina1911.15.17 do mesmo modo que a determinação da porcentagem de formas espermáticas anormais 3.10.16.18.19. Tais características foram ainda relacionadas com as estaçōes do anols e com a frequência das ejaculaçôes 19.

Alguns autores correlacionaram o volume do ejaculado ao peso vivo do animal6,13, enquanto outros verificaram uma relação inversa entre volume e concentração espermátical 10.23 .

A intluência do pH e da pressão osmótica sobre a motilidade espermática também foi investigada em diferentes diluidores específicos para cães 7,8 .
Por outro lado, características bioquímicas do sêmen foram estudadas2, verificando-se o eféto deletério de diluidores hipotônicos 20 e o aumento da motilidade espermática pelo potássio, magnésio e cálcio2!. bem como a concentração de sódio e potássio nas três frações do ejaculado.

Na referência disponivel sobre a raça Pastor Alemãolt, foi investigado o número total de espermatozóides por ejaculado e a motilidade espermática, objetivando a prática de inseminação artificial com sêmen congelado.

Diante desta escassez, objetivou-se o estudo de caracteristicas físicas, morfológicas e da concentração de sódio e potássio do sêmen de cães da raça Pastor Alemão, frente a quatro diluidores, para utilizaçāo em inseminação artificial.

\section{MATERIAL, E MÉTODO}

Na pesquisa foram utilizados 25 cães da raça Pastor Alemão, com idade de 1 a 7 anos, pesando de 30 a 35 quilos e pertencentes ao Canil da Polícia Militar do Estado de São Paulo. Os animais, submetidos diariamente a exercícios de treina- 
mento, recebem alimentação básica de carne cozida. arroz. cenoura e leite. kendo sido selecionados após exame clínico geral e específico do aparelho reprodutor e após adaptação ao mélodo de masturbação para colheita de sêmen, foram individualmente catalogados.

As colheitas foram efetuadas pela manhã, antes dos exercícios diários e pelo menos 48 horas após coberturas. Cada animal forneceu 5 ejaculados, com intervalos de uma semama entre colheitas. sendo colhidas a segunda e parte da terceira fração em tubo de ensaio mantido em recipiente térmico ${ }^{3}$, verificando-se de imediato o volume, a cor e o aspecto do material seminal, a seguir colocado em banho-maria a $37^{\prime \prime} \mathrm{C}$, para posterior avaliaçẫo da motilidade em microscopia comum, com resultado expresso em porcentagem e o vigor da motilidade calculado em escala de 0 a 5.

O pH foi determinado em pHmetro*, a pressão osmótica em osmômetro** e a quantilicação de sódio e potássio através de fotômetro de chama***. A concentração de espermatozóides por $\mathrm{mm}^{3}$ loi verificada pelo método hematimétrico, em câmara de Neubauer, servindo de base para o cálculo do número de células espermáticas no cjaculado.

A morlologia espermática foi estudada em lâminas coradas pelo método de Williams e em preparações úmidas em microscopia de interferência diferencial sob imersão e aumento de 1.250 vezes. A porcentagem de formas anormais foi estabelecida pela contagem de 200 células espermáticas, classificando as patologias em defeitos maiores e menorest.

Quatro diferentes diluidores. preparados 24 horas antes de iniciadas as colheitas. foram caracterizados quanto ao pH. à pressão osmótica (mOsmois) e à concentração de sódio e potássio (mŁig/l).

D) (iema-citrato

Solução de citrato de sódio a $2.94 \% \ldots \ldots \ldots \ldots .80$ ml

$\mathrm{D}_{2}$ (iemal-lactose

Solução de lacrosc a $11 \% \ldots \ldots \ldots \ldots \ldots \ldots$. . . . . . .

$\mathrm{D}_{3}$ Gema-leite desmatado

Solução de leite em pó desnatado a $9 \% \ldots \ldots \ldots . .80 \mathrm{ml}$

I) TRIS-gema

Frulose
TRIS

Ácido cítrico $1.30 \mathrm{~g}$

$\mathrm{H}_{2} \mathrm{O}$ $.72 .20 \mathrm{ml}$

A cada um dos diluidores foram acrescentados $20 \mathrm{ml}$ de gema de ovo, $1000 \mathrm{UI}$ de penicilina/ml e $1000 \mathrm{\mu g}$ de estreptomicina/ml.

Para descrição dos resultados foi utilizada a Análise de Variância para estimar a média populacional de cada variável. comparar a variabilidade entre colheitas de sêmen de um mesmo animal e estimar as correlações existentes entre as variáveis estudadas. Estimativas por Ponto das Médias e Comparações Múltiplas pelo Método de Tukey foram feitas para comparar os 4 diluidores relativamente à sua influência no pH e pressão osmótica do sêmen.

\section{RESULTADOS}

As médias gerais, assim como os valores médios, mínimos e máximos de cada característica estudada no sêmen de cães da raça Pastor Alemão, podem ser observados ná Tab. 1.

\section{TABELA 1}

Médias gerais e valores mínimos e máximos das médias de características seminais de 25 cães da raça Pastor Alemão. São Paulo. 1986.

\begin{tabular}{|c|c|c|c|}
\hline Característical & Média & Valor mínimo & Valor máximo \\
\hline Volume (ml) & 7.17 & 2.70 & 13.80 \\
\hline Motilidade (\%) & 68.0 & 0.0 & 90,0 \\
\hline Vigor (0-5) & 3.55 & 0.0 & 4.0 \\
\hline \multicolumn{4}{|l|}{ Concentraçào } \\
\hline Espr/mm & 136.192 & $18.2(0)$ & 326.880 \\
\hline \multicolumn{4}{|l|}{ Espz/cjaculado } \\
\hline$\left(x \mid 10^{3}\right)$ & 885.772 & 59.150 & 1.689 .424 \\
\hline $\mathrm{pll}$ & 6.32 & 6.02 & 6.60 \\
\hline \multicolumn{4}{|l|}{ Pressão osmóti- } \\
\hline ca (mOsmois) & 289.20 & 285.20 & $295 .(x)$ \\
\hline Sódio (mEq/l) & 145.32 & 1.34 .40 & 156.00 \\
\hline Potássio (mEq/l) & 11.06 & 7.80 & 13.72 \\
\hline
\end{tabular}

Espz = espermatozóides

\footnotetext{
* = Radiomeler $\mathrm{BMS}_{3}$ MK, Blood Micro Cister

*** = IISKE OMm OSMOMETTER

*** = I)MIC - I DI(jIME.I)
} 


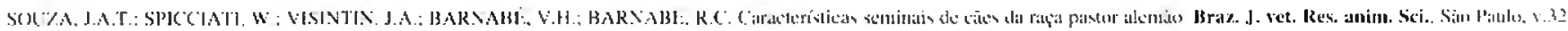

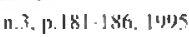

A somatória das médias dos defeitos espermáticos maiores e menores apresentou valores mínimos, máximos e total geral representados na Tab. 2.

TABLIA 2

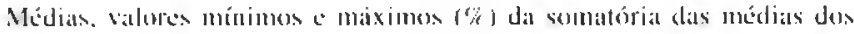

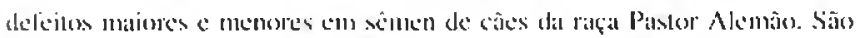
P'itulo, 19x6.

\begin{tabular}{|c|c|c|c|}
\hline Tolial & Médial & Valor mínimo & Vaslor máximo \\
\hline Deforim matiores & 9.92 & 3.25 & $2.3,40$ \\
\hline Deleitum menores & 7.62 & 1.10 & 27.80 \\
\hline Gival & 17,52 & $+.7(1)$ & 51.20 \\
\hline
\end{tabular}

O pH. a pressão osmólica ce al concentraçẫo de sódio e de potássio dos quatro diluidores estão mencionados nal T'ab. 3.
TABELA 3

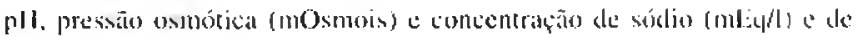
polassio (mF P'atuls. 1986

\begin{tabular}{|c|c|c|c|c|}
\hline $\begin{array}{l}\text { Carateristicas } \\
\text { Diluideres }\end{array}$ & pH & P. osmoticat & Sóntion & P'olíssion \\
\hline Giomia-citralto & 6.90 & 200 & 270 & 6.8 \\
\hline Gena lidelone & 0.25 & 310 & 3 & 0,0 \\
\hline Gomal-kitc & $0,40)$ & 240 & 10 & 25.0 \\
\hline TRIS-g넨 & 5.85 & 300 & 4 & 0,0 \\
\hline
\end{tabular}

mosmors = miliosmois

mligfl = milcyuis'alente por titro

A Tah. 4 repecsenta as correlaçoes ented at calracteristicas seminais de cãces da raça Pástor A lemão.

TABEASt

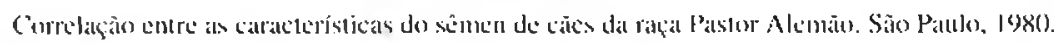

\begin{tabular}{|c|c|c|c|c|c|c|c|c|c|c|c|c|c|c|c|c|c|c|}
\hline Vandets. & Violume & $\begin{array}{l}\text { Mont- } \\
\text { dade }\end{array}$ & Viger & $\begin{array}{l}\text { Concen- } \\
\text { traçais' }\end{array}$ & $\mathrm{pH}$ & $\begin{array}{l}\text { P.Osno } \\
\text { tita }\end{array}$ & $\mathrm{Na}$ & K & Idate & $\begin{array}{l}\text { Calbęa } \\
\text { idmil }\end{array}$ & $\begin{array}{l}\text { P. Int } \\
\text { idrai }\end{array}$ & $\begin{array}{l}\text { Cicuda } \\
\text { idmi }\end{array}$ & $\begin{array}{c}\text { G.C.D. } \\
\text { (dm) }\end{array}$ & $\begin{array}{l}\text { AcNo } \\
\text { somo } \\
\text { (DM) }\end{array}$ & $\begin{array}{l}\text { Cabcya } \\
\text { (D)I }\end{array}$ & $\begin{array}{l}\text { P. Pea } \\
\text { (D) }\end{array}$ & 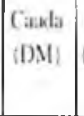 & $\begin{array}{l}\text { (GCP } \\
\text { (DM) }\end{array}$ \\
\hline \multicolumn{19}{|l|}{ Voluturk } \\
\hline Motlidade & $14 \times 2$ & & & & & & & & & & & & & & & & & \\
\hline Vigor & 11331 & $N(6) 1$ & & & & & & & & & & & & & & & & \\
\hline Concentą̧äs & -36.14 & 3873 & .468 & & & & & & & & & & & & & & & \\
\hline $\mathrm{pH}$ & -.2531 & .2339 & -1114 & .164041 & & & & & & & & & & & & & & \\
\hline P. O-móxica & -1205 & an?2 & -11361 & 0184 & (Ix)22 & & & & & & & & & & & & & \\
\hline $\mathrm{Na}$ & .1262 & $(460)$ & -0.3557 & -.778 & -2199 & $-2 \times 61$ & & & & & & & & & & & & \\
\hline k & .0774 & -1890 & $-238 x$ & $-(1602$ & -1791 & -1009 & .1000 & & & & & & & & & & & \\
\hline Whake & .1578 & +4037 & -3931 & $-52 \times 2$ & -.1526 & -0020 & .2483 & . 1820 & & & & & & & & & & \\
\hline Cabequa Intay & $(W \%)$ & -.7670 & -7736 & -2902 & .3233 & -01551 & -.11 .45 & 3209 & 4264 & & & & & & & & & \\
\hline P fintemed idm) & -.0665 & 3432 & $-.10+5$ & -1615 & (28)50 & -11639 & 1529 & 0389 & 0776 & .2718 & & & & & & & & \\
\hline Cauda idmi & -19978 & -5121 & -3884 & -1433 & .2236 & $(x) y ?$ & -1707 & $-10(2) 5$ & .2615 & +1924 & $.157 \%$ & & & & & & & \\
\hline G.CD) (dm & $-0: 03$ & $-514 ?$ & $314 i$ & -.0927 & 1335 & .1834 & $-135 i$ & 1069 & .1958 & $549 ?$ & $+1+10$ & $31 \times x$ & & & & & & \\
\hline Acrossono idnal & .1162 & -.2314 & -.1107 & -.1158 &.$\% 3$ & $-(690)$ & -.11115 & .1516 & 1313 & .1944 & .2670 & 8179 & 1796 & & & & & \\
\hline Cabcẹa (ún) & $-1132 x$ & -54018 & -6218 & $-(1893$ & .3145 & 1403 & -6715 & $.26+17$ & .2585 & .7319 & .2859 & 3331 & 3226 & 0955 & & & & \\
\hline PInterw (úm) & 10301 & $-4(0) 1$ & -2683 & -.1593 & $-01 \times 02$ & .0093 & $-.056 ?$ & .19984 & .1817 & 2961 & .3336 & 2688 & $.488 \%$ & .2566 & .1273 & & & \\
\hline Crub idm) & -0374 & -.5233 & -3623 & $-.220 x$ & .1569 & 1312 & -.1633 & -0448 & .1918 & 4217 & .2471 & 6075 & .4595 & 0959 & 1970 & $36+6$ & & \\
\hline GC.P. IImI & $-(X), 98$ & -6713 & -.4590 & -114 & $.157 x^{1}$ & $-(1320$ & -.0361 & $2(x) 3$ & .2240 & $5 \times 19$ & $5(\%)$ & 3353 & .4150 & 3162 & $415 \mid$ & $.5141^{1}$ & $3(15)$ & \\
\hline
\end{tabular}

$\mathrm{DM}=$ defeitos maiores $\mathrm{dm}=$ deteiton nemores 


\section{DISCUSSTO}

Dentre os vários mélodos de colleita de sêmen no caio, a

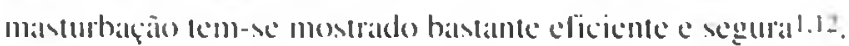

() ejaculado do cio compoesese de 3 fraçoes distin-

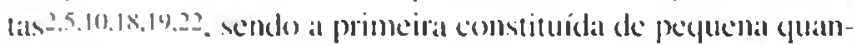
tidake de liquido clano e transparente. com ou sem experma-

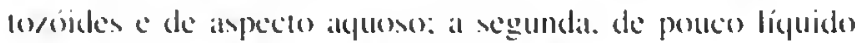

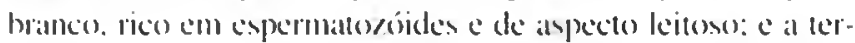
ceira, de liquido claro ou tranyparente. praticamente desprovidat de espermatozódes e de alspecto alyuoso, representando o maior volume do cjaculador.15. Nín comsideramos o ejaculat-

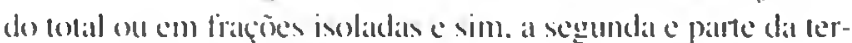

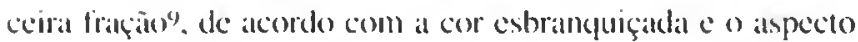
keitoso. que estäo relacionados com a concentração espermáticall. com o intuito de efetuar diluçãon e comservaçăo destal proçäo rica em espermatosóides. e alender ate sentido prático da inseminaçäo artificial $1+172021$.

O volume da segunda c parte da terceira fraçäo seminal variou. em médial. de 2.70 a $13.80 \mathrm{ml}$ (Tabela 1). tendo como média populacional $7.17 \mathrm{ml}$. não podendo. portanto. ser compalrado com on resultados dos atutores que traballharam com o ejaculado lotal ${ }^{2}, 10.13,16.10 .23$ e em 3 fraçoes isoladamente.5.11.15.18. Vão obstante. os resultados saio superiores aqueles que comsideraram a porção ricat em esperma[oróides"s?

Emborat a motilidade progersiviva retilínca não constitua. por si só. padrão de medida de vitalidade e poder de fecundação dos espermatoródes. ela é necessíria parat at fertilização. tornando-se, ention, importante partintetro paral avaliaçào imediata do sêmen. A motilidade variou. em médiat. de 0.0 a $90.0 \%$ (Tab. I). encuanto a média populacional foj de $68.8 \%$. estando de acordo. tanto para os ejaculados totatis quanto para on fracionados 17.19. porém inferior a on$\operatorname{tros} 3.9,14.15 .18$

Apesall de nenhum dos autores comsulados ter classificado als palologias em defeiton maiores e menores. constaltamos que o total de espermatoröides anormais (Tab. 2) encontra-

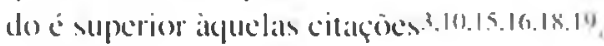

A média de pH. 6.32. com variaçăo de 0.02 a 6.60). entá proxima as dos aulores yue comsideraram o cjaculado fracionado17.22. onde podemos observar gue a lereciral traf̧aos ou fraçäo prostáticat possui certa influênciat tampão no çaculado total5,20, Já palta o cjalculado total ocerre. ocasionalmente. semelhançals ou ligecira interioridadell 16.23. Devemos salienlat que o pH pode variar com a riqueza espermática. ou seja, o sêmen rico em espermalozöides apresenta pll ligeiramente ácido e. quando pobre. ligeiramente alcalinol.20. podendo ainda dar uma idéiat da concentraçäo eypermáticall
() pH dos quatro diluidores a deskes. yuando acrescidos de sêmen. mostrou uma ligeiral variaçäo, sendo yuc os diluidores 1), e 1); mais o sêmen mostranam pH médio superior à média gèal do sêmen puro. Jí o sêmen diluíclo em D) c D, mostrou pll médio inferior. Pelas comparaçóes múltiplas (dovis a dois). verificou-se yue os yuatro diluidores angem diferentemente sobre o pll do sêmen. Na liferatura encontrase afirmaleajo de que os espermatoroides posstem melhor sobrevivência em diluidores gema-lampäo compll 6.678.

A prensiono osmótica do sêmen apresentou media de 289.20 e limites de 28.5 .20 a 295.00 mosmois (Tath. 1). valores que não mostraram variação significante entre os animais estudados, o mesmo náo ocorrendo com os diluidores. Os diluidores $D_{1}, D_{2}$ e $D_{4}$ e D $D_{3}$. (quando acrescidos do sêmen. apresentaram pressiáo osmótical média respectivamente superior e inferior à média geral no sêmen puro. () efeito deletério sobre as células espermáticas, dos diluidores hipononicos em comparação anos hipertônicos já foi salientado?on. mesme yuando de composiçán similar. A conicidade dos diluidores é comsideradda obima quando o FPPI) (Freesing Point Depression) silua-se contre -.52 a $-.66^{\circ} \mathrm{C}^{\mathrm{N}}$. 0 yec concorda com os resultados obtidos. Além disso. comstatou-se yue os espermatozóider loleram mais as variaģöes de tonicidade no diluidor TRIS-gema do que no citratogemal. emboral a pressiano osmóti(a medida pelo IFPI) scja mais alta no TRIS-gema?

As concentraçoes médias de súdio e potíssio no sêmen foram. expectivameme. 14.5.32 ml:y/l $(1,34.40$ a 1.56 .00$)$ e $11.06 \mathrm{ml}: \mathrm{d} / 1$ (7.80) a 1.3.72) (Tiab. 1). Considerando o e jaculado fracionado. os valores são concordantes com a literaturia2.5.2.2.

A Tab. 3 montrat a concentraçäo de sódio e potíssio nos diferentes diluidores. gemat-citrato 270.0 e 6.8: gema-lactose

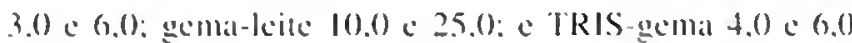
mF.y/l. Jí foi verificado yue potássio, magnésio e cálecio, isoladamene nos dibuidores. atumentam a motilidade dos espermallocóides. encyuanto o efeito dos três combinados é menor do que potássio e magnésio ou potássio e cálcio juntos. salientando-se aindal yue o excesso de polássio deprime a motilidade dos espermatoróideszon.e.

Verificamos, ainda. yue houve variaçoes significantes entre caies para lodas as calracteristicas seminatis estudadals. excelo no que se refere à premio osmónica. Na Tah, 4 verificam-se as correlaçoes entre as diferentes calracteristicals seminais. observando-se forte correlaçäo positiva entre a motilidade e o vigor, entre os defeitos individuais maiores a menores de cabeça. de cauda e gotas citoplasmáticas além de acentuala correlaçäo negativa da motilidade e vigor com as anormalidades espermáticans individuais de cabeça e gota citoplas. mátical proximal. 


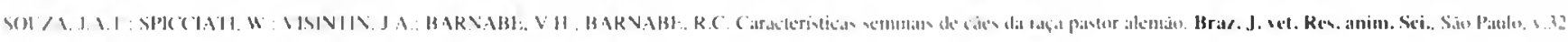

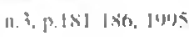

\section{CONCILLSÕES}

() estudo de algumas carancterísticas lísicals. morlobógicals e bioguimicas do simen de cièes da raça Pastor Alemaio permile as seguintes conclusies:

I - A variabilidade das caltacterísticas entre càes é bem maior em relaçàn a variabilidade entre as colheitas de cada annimal. excelo parat a pressão oxmótical e concentraţão de sodio yue apresentam variabilidade maior entre as colbeitas de um mesmo animal do que entre os diferentes cates.

2- A motilidade apresentat boal correlação positiva com o

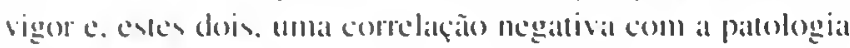
clill geral.
3 - As amostras de sêmen diluídas em gema-citrato e gemalleite desnatado mostram médias de pH mais altats em relação à média geral observada no sêmen puro. encyuanto as amostras diluidas em gema-lactone e TRIS-gema apresentam médias mais balixar: pelas comparaçóes múleiplas (dois a dois). os quatro diferemtes diluidores agem diferentemente sobre opll.

4- As amosmass de sêmen diluidas cm gemat-citrato. gemalactose e TRIS-gema apresentam médian de presiono osmótica mais altas cm relação a média getal observada no sèmen puro. emguanto als amostras diluídas em gema-leite desnatado revelam média mais baixa: pelas comparaçōes múltiplas (dois a dois). on diluidores gema-lactose e TRIS-gema agem de forma igual sobre a pressão osmótica.

\section{SIMMARY}

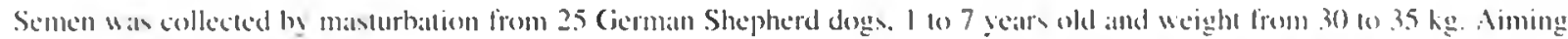

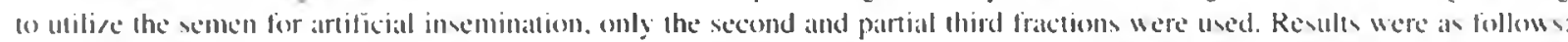
meall cjaculation volume $7.17 \mathrm{ml}$ : white colour and milky aspect: sperm motility $68.84 \%$ : vigour 3.5 : concentration 1.36 .192

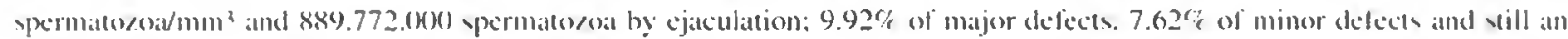
individual ahnormalition classification. The pll of semen. of extenders and of extended semen. respectisely. varied from 6.02

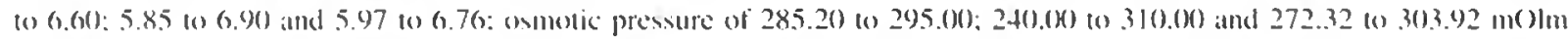

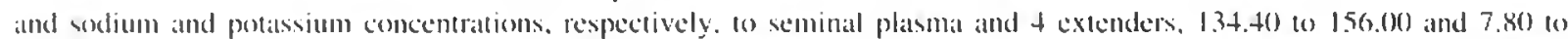

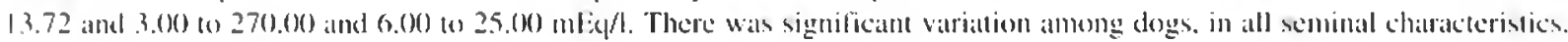
exceph for commotic pressure. There was also conrelation between the seminal characteristics and between these and cotal sperm abnormalities. Varialvility of seminal characterivices among dogs is greater when comparing collections of each animal. with exception of usmotic pressure and sodium concentration variability that is reversed.

UNITERNIS: Semen: (ierman Shepherd doge

\section{REFERÊNCIAS BIBLIOGRÁFICAS}

1-A.VTOIN\&: I. Contribution à l'étude de l'insémination artificielle chez le chien: élude systématique du yerme de chicen. Alfort. 1952 (These pour le docloral). I:cole Nationale Vétérinaire.

2-BARTI.1:1"I. D.J. Biochemical characteristices of dog semcon. Nature. 1.182, p. 1005-6. 1958.

3-BICUDO). S.D. I:vludo de algumats caratcterísticats do émell do cato. In: SIMPÓSIO NACIONAL DH: R1:PROD(C) AOVIMAL. 5.. Anais. Belo Horizonte. p..395-6. 198.3.

4-B1.O.M. E. Ultratstrukturen af nogle harakteristike spermicdefekter og forslag til el nyt klassificerings-system for ly rems spermiogram. Flhe ullatstructure of some chatacteristice sperm defects and a proposal for a new classificaltion of the bull spermiogram/. Nordisk Veterinärmedicin. v.25. p. 38.3-91. 197.3

5-BRAOU I) : P. Contribution à l'étude de l’insémimation arfificielle dans l'espèce canine. Alfort. 1979) (Thèse pour le doctorat). Ecole Nattomale Vélérinaire.

6-DLBII:L. A. Studies in semen collection by masturbation method and on ejaculation reflex in doges. Weterynaria Wroclaw, 1.29, p. 22.5-34, 1972.

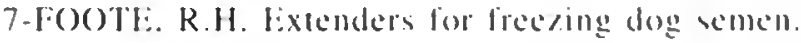
American Journal of Veterinary Kesearch, v.25, p.37-40. 1964.

8-FOOTl:. R.H.: I.EONARD. E.P. The influence of pH, osmotic pressure, glycine, and glycerol on the survival of 


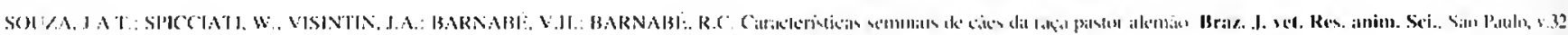

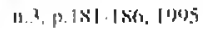

dog sperm in buffered-yolk extenders. Cornell Veterinarian, v.54. p. $78-88.1964$

9-(ill.L. H.P.: KAUFMAN, C.F.: FOOTE. R.H.: KIRK. R.W. Artificial insemination of beagle bitches with treshly collected. liquid-stored. and fromen-stored semen. American Journal of Veterinary Rescarch. v.31. p.1807-13. 1970.

I0-HANCOCK. J.L.: ROWLANDS. I.W. The fisiology of eproduction in the dog. Veterinary Record. v.47. p.7719. 19-49.

11-HARROP. A.E. Some observations on canine semen. Veterinary Record. v.67. p.494-9. 195.5.

12-KIRK. R.W. Artilicial insemination in the dog. Allied Veterinarian. v.30, p.40-4. 1959.

13-LAURA.VS. R.: WII.C\%YNSKA. H. Algunas obscrvaciones sobre el volumen, la concentración y la conservación del esperma normal de perro. Revista de Fisiopatologia e Reprodución Animal. Madrid. v.2. p.736. 1949.

14-1.IES. G.E.: CASTLEBERRY. M.W. The use of fromen semen for artificial insemination of German shepherd dogs. Journal of the American Animal Hospital Association, v.13. p.382-6. 1977.

1.5-MIAL.OT. J.P. Examen de l'appareil genital du chicn. Point Vétérinaire. v.10. p.65-72. 1980.

16-PI:RI:Z GARCIA. T.P. Aportaciones a los mélodos de recongeda y contrastación del esperma de peror. Revista del Patronato de Biologia Animal. v..3. p.97-1.38. 1957.
17-ROYCHOUDHURY, P.N.: I)LBA). M.L. Observations on dog semen. I. Dilution and conservation in three extenders. Zootecenica e Veterinaria. Milano, 1.29. p.117-21. 1974.

18-TAHA. M.B.: NOAKI:S. 1).1:.: ALLE.V. W.I:. The aflect of season of the year on the chatackeristics and composition of dog semen. Journal Small Animal Practice. ง.22. $1.177-84.1981$.

19-TAHA. M.B.: V()AKES. 1).1:. NI.LE.N. W.1:. The effect of the frequency of ejaculation on seminal charackristices and libido in the beangle doge. Journal Small Animal Practice. v.24. p.309-1.5. 1983.

20-WAI.ES. R.G.: WHTHE I.G. The interation of $\mathrm{pH}$ conicity and electrolyte concentration on the motility of don spermatozoat. Journal of Physiology. I ondon, v.1+1, p. 27.3 80.1958

2I-WAL.F.S. R.G.: WHIT': I.(i. The eflect of the iom of the alkali metals magnesium and calcium on dog speimalozoa. Journal of Physiology. I.ondon. $1.1+2$. p.494-502. 1958.

22-W ALES. R.(i.: WHITE. I.G. SomL observation on the chemistry of dog semen. Journal Reproduction and Fertility. 1.9. p.69-77.196.7.

23-WHITE, I.G. Biochemical aspeces of mammalian semen. Animal Brecding Abstracts. v.26. p. 109-2.3. 1958.

Receloido para publicação em 19/08/93 Aprovado para publicação em 13/12/94 